\title{
Early neurological impairment and severe anemia in a newborn with Pearson syndrome
}

\author{
Anne-Sophie Morel • Nadia Joris • Reto Meuli • \\ Sébastien Jacquemont • Diana Ballhausen • \\ Luisa Bonafé • Sarah Fattet • Jean-François Tolsa
}

Received: 6 November 2007 /Revised: 29 April 2008 / Accepted: 2 May 2008 / Published online: 14 June 2008

(C) Springer-Verlag 2008

\begin{abstract}
Background Pearson marrow-pancreas syndrome (PS) is usually a fatal mitochondrial disease, mostly diagnosed during infancy or postmortem. PS is caused by the deletions or duplications of mitochondrial DNA (mtDNA). The tissue distribution and relative proportions of expressed abnormal mtDNA determine the phenotype and the clinical course.

Materials and methods We describe the case of a term baby boy who was diagnosed with PS early in the neonatal period due to severe aregenerative anemia and persistent lactic acidosis.

Results His neurological examination was abnormal since birth. Brain magnetic resonance imaging (MRI) at term was abnormal, indicating that mitochondrial encephalopathy in
\end{abstract}

A.-S. Morel $•$ N. Joris $\cdot$ J.-F. Tolsa $(\square)$

Division of Neonatology, Department of Pediatrics,

University Hospital and University of Lausanne, CHUV,

1011 Lausanne, Switzerland

e-mail: Jean-Francois.Tolsa@chuv.ch

R. Meuli

Department of Radiology,

University Hospital and University of Lausanne,

1011 Lausanne, Switzerland

\section{S. Jacquemont}

Division of Genetics, Department of Gynecology and Obstetrics,

University Hospital and University of Lausanne,

1011 Lausanne, Switzerland

D. Ballhausen $\cdot$ L. Bonafé

Division of Molecular Pediatrics, Department of Pediatrics,

University Hospital and University of Lausanne,

1011 Lausanne, Switzerland

S. Fattet

Division of Onco-Hematology, Department of Pediatrics,

University Hospital and University of Lausanne,

1011 Lausanne, Switzerland
PS can be already manifested in the neonatal period. To our knowledge, neonatal encephalopathy in PS has not been previously described.

Conclusion PS is a rare condition diagnosed in the newborn. It should be suspected in the presence of severe anemia and persistent lactic acidosis, and may manifest with early encephalopathy.

Keywords Pearson syndrome.

Mitochondrial encephalopathy - Aregenerative anemia .

Cerebral lesions · Brain MRI

$\begin{array}{ll}\text { Abbreviations } \\ \text { ADC } & \text { Apparent diffusion coefficient } \\ \text { KSS } & \text { Kearns-Sayre syndrome } \\ \text { MRI } & \text { Magnetic resonance imaging } \\ \text { MRS } & \text { Magnetic resonance spectroscopy } \\ \text { mtDNA } & \text { Mitochondrial DNA } \\ \text { PLIC } & \text { Posterior limb of the internal capsule } \\ \text { PS } & \text { Pearson syndrome }\end{array}$

\section{Introduction}

Pearson marrow-pancreas syndrome (PS) is a rare multisystemic mitochondriopathy with lactic acidosis and nonneuromuscular initial expression involving the hematopoietic tissue, the exocrine pancreas, the liver, and the kidneys [7]. The typical clinical course is characterized by progressive liver failure and intractable metabolic acidosis, with death in infancy. Survivors later develop neurological symptoms, such as in Kearns-Sayre syndrome (KSS) [6].

PS is frequently diagnosed in early infancy, but rarely during the neonatal period. Prenatal hematological manifestations of PS have also been described [3]. The cause of 
the disease is most often de novo deletions or, more rarely, duplications in the mitochondrial DNA (mtDNA). Largescale duplications coexist, with deletions in some cases. The diagnosis is confirmed by the Southern blot analysis of white blood cells, which identifies mtDNA rearrangements. The severity of the clinical manifestations and the rate of disease progression are variable and correlate with the mtDNA gene mutation load [9] and the distribution of mutation expression [4]. Currently, only supportive treatment is available.

We present the case of a child with PS diagnosed early in the neonatal period, including a detailed description of the neurological picture, brain magnetic resonance imaging (MRI) scans and clinical follow-up until death at 18 months of life.

\section{Methods and results}

\section{Case report}

A full-term boy was born after an uneventful pregnancy to healthy unrelated parents of Swiss and North African origin. His mother had chronic hepatitis $\mathrm{C}$ with no detectable viremia and received adequate prenatal care. The baby presented intrauterine growth restriction, with birth weight $2,360 \mathrm{~g}(<$ percentile 10), length $47 \mathrm{~cm}$ (<percentile 10), and head circumference $35.5 \mathrm{~cm}$ (percentile 10-50). The family history was unremarkable; a 4-year-old sibling is healthy. Emergency cesarean section was required for pre-eclampsia and altered cardiotocogram. The Apgar score was 6/7/8 and cord blood pH 7.17 and 7.27 (arterial and venous, respectively). Following birth, the baby presented with extreme pallor, a loud cardiac murmur, hepatomegaly, hyporeactivity, and hypotonia. Within one hour after birth, in the setting of severe anemia, he developed tachypnea and lactic acidosis, without hypoglycemia or ketosis, consistent with a diagnosis of cardiogenic shock. He required blood transfusions, pressure support, and bicarbonate infusion, followed by endotracheal intubation at 12 hours of life.

The first laboratory results at 1 hour of life showed aregenerative microcytic anemia (hemoglobin $29 \mathrm{~g} / \mathrm{l}$, normal range 135-215) and severe lactic acidosis (blood lactate $14 \mathrm{mmol} / \mathrm{l}$, normal range $0.63-2.44$ ) in the absence of hypoxia. An early echocardiography on day 1 of life showed signs of chronic antenatal hypoxia with dilatation of the coronary arteries and right cardiac hypertrophy. After stabilization, the patient was extubated on day 3 of life. Asymptomatic thrombocytopenia (thrombocytes 48 G/l, normal range 150-350) associated with persistent aregenerative anemia were noted on day 5 . We also observed a persistent lactic acidosis (venous lactate $3-5 \mathrm{mmol} / \mathrm{l}$, normal range $0.63-2.44)$. Neurological examination revealed an irritable baby unable to suck or swallow, with a marked axial hypotonia and severe head lag. Peripheral tone was normal, archaic reflexes were present, but no deep tendon reflexes could be elicited. No tremor was noted. At 2 weeks of life, the patient presented with post-prandial vomiting without signs of malabsorption (fecal elastase within the normal limits for age). Liver and pancreatic tests were normal. Gastroesophageal reflux was suspected and the symptoms spontaneously improved with antireflux positioning and nasogastric feeding.

At birth, a cerebral ultrasound showed periventricular echogenicities and a right choroid cyst. Brain MRI, performed at 10 days of age (corrected age, 40 weeks), demonstrated no cerebral congenital malformations and no hemorrhage. Spectroscopy was abnormal, with increased peak of lactate in the posterior white matter, but no other abnormal metabolites (Fig. 1). Moreover, diffusely excessive high signal intensity on $\mathrm{T} 2$-weighted images were noted in the white matter (Fig. 2), with increased values $\left(19 \times 10^{-5} \mathrm{~mm}^{2} / \mathrm{s}\right.$, normal range $15-16 \times 10^{-5} \mathrm{~mm}^{2} / \mathrm{s}$ ) on apparent diffusion coefficient (ADC) maps. In addition, the posterior limb of the internal capsule (PLIC) showed an abnormally low level of myelination on inversion recovery T1 images (Fig. 3).

Extensive investigations were performed for the severe anemia. The placental examination showed no sign of abruptio or torn vasa praevia. Trans-placental hemorrhage was excluded by a negative Kleihauer-Betke test. Acute internal hemorrhage was ruled out with normal cerebral and abdominal ultrasounds. There was no hemoglobinopathy, no hemolysis, nor jaundice. Coombs' test was negative and glucose-6-phosphate dehydrogenase activity was normal. Infectious screening was negative for parvovirus B19, cytomegalovirus, Epstein-Barr virus, malaria, and syphilis. Hepatitis $\mathrm{C}$ screening was positive for $\operatorname{IgG}$ (60 times the norm) but negative for hepatitis $\mathrm{C}$ antigen, consistent with maternal infection. On day 4 of life, metabolic investigations showed elevated Krebs cycle metabolites in the urine: fumarate $174 \mathrm{mmol} / \mathrm{mol}$ creatinine (normal range 4.2-21.9), malate $252 \mathrm{mmol} / \mathrm{mol}$ creatinine (normal range 10.8-47.8), 2oxoglutarate $423 \mathrm{mmol} / \mathrm{mol}$ creatinine (normal range 58.7 338.6), and lactate $1,327 \mathrm{mmol} / \mathrm{mol}$ creatinine (normal range 25.9-91.9). Lactate was also elevated in the cerebrospinal fluid $(5.53 \mathrm{mmol} / \mathrm{l}$, normal range $<1.8 \mathrm{mmol} / \mathrm{l})$, raising the suspicion of a mitochondrial disorder. Bone marrow aspiration and genetic testing were performed on day 19 of life. Bone marrow smear demonstrated a sideroblastic anemia with vacuolated erythroblasts, the hallmark findings for PS [1]. This diagnosis was confirmed by the Southern blot study of the mtDNA, which revealed a $4.7-\mathrm{Kb}$ deletion, with $60 \%$ heteroplasmy in blood and bone marrow leucocytes.

At 1 month of life, the patient developed pancytopenia with anemia requiring several blood transfusions, leucopenia, and transient agranulocytosis. By 2 months of life, the 
Fig. 1 A magnetic resonance imaging (MRI) spectroscopic acquisition demonstrated a normal level of choline, creatine, and $\mathrm{N}$-acetyl-aspartate. A mild signal of lactate is observed in the posterior white matter (arrow)

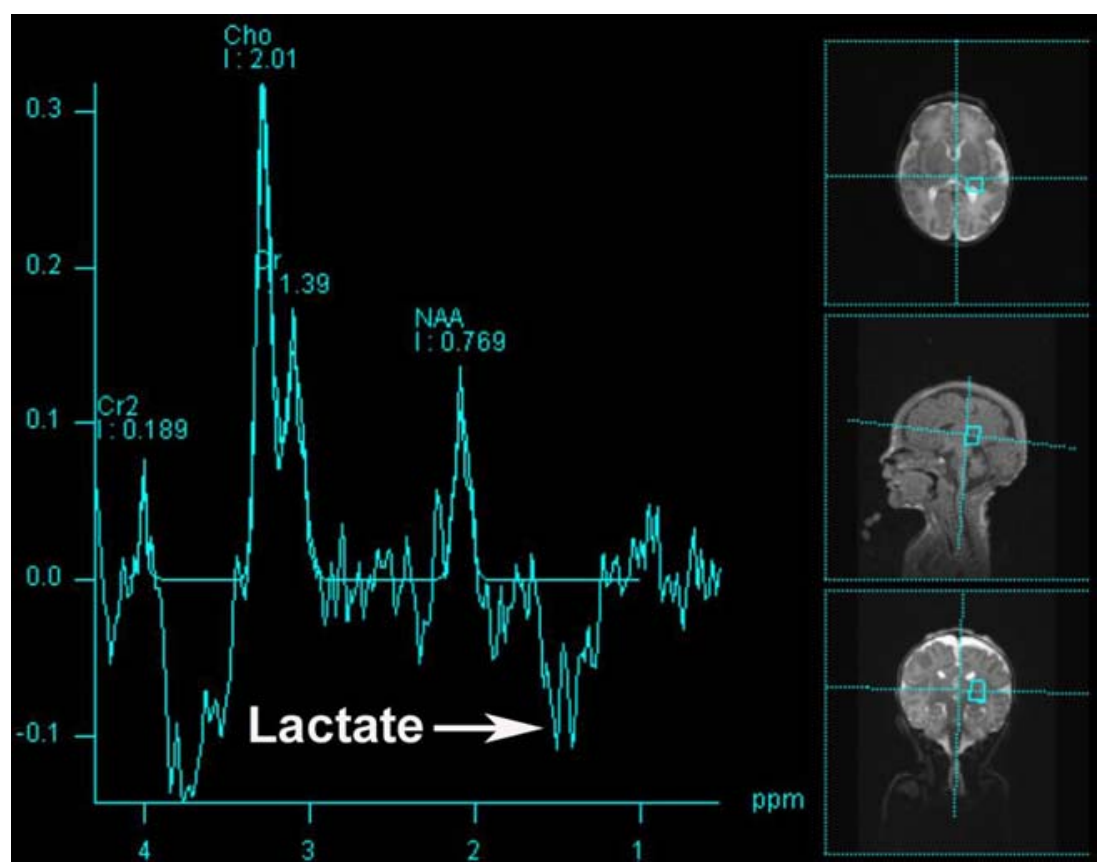

difficulty in sucking and swallowing had resolved spontaneously. The neurological status remained pathological, with axial hypotonia being the most prominent clinical feature. The baby was discharged with the implementation of a multidisciplinary network of care and family support. Follow-up until 1 year of age was characterized by persistent failure to thrive (weight $<$ percentile 3; length at percentile 3; head circumference at percentile 50-75) and by several hospital admissions due to viral infections (gastroenteritis, Varicella-Zoster infection), as well as bacterial pneumonia.

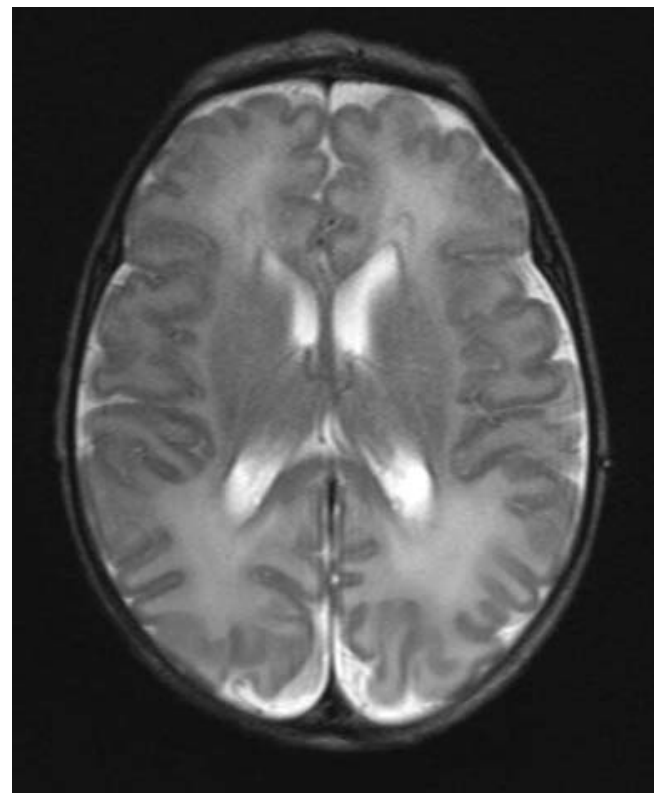

Fig. 2 T2-weighted transverse image at the level of the upper part of the lateral ventricules shows a diffusely excessive high signal intensity in the white matter
Anemia was corrected with erythrocyte transfusions every three weeks. Moderate to severe neutropenia (absolute neutrophile count $0.5-1.5 \mathrm{G} / 1$, normal range 1.0-9.0) and moderate thrombocytopenia (thrombocytes 45-100 G/1, normal range 150-350) persisted. Urinary organic acids showed persistent increased excretion of lactate and Krebs cycle intermediates. Chronic lactic acidosis required oral bicarbonate treatment. Orotic acid was measured at least three times and was never elevated (maximum value $0.96 \mathrm{mmol} / \mathrm{mol}$ creatinine, normal value $<3.3$ ). No diabetes

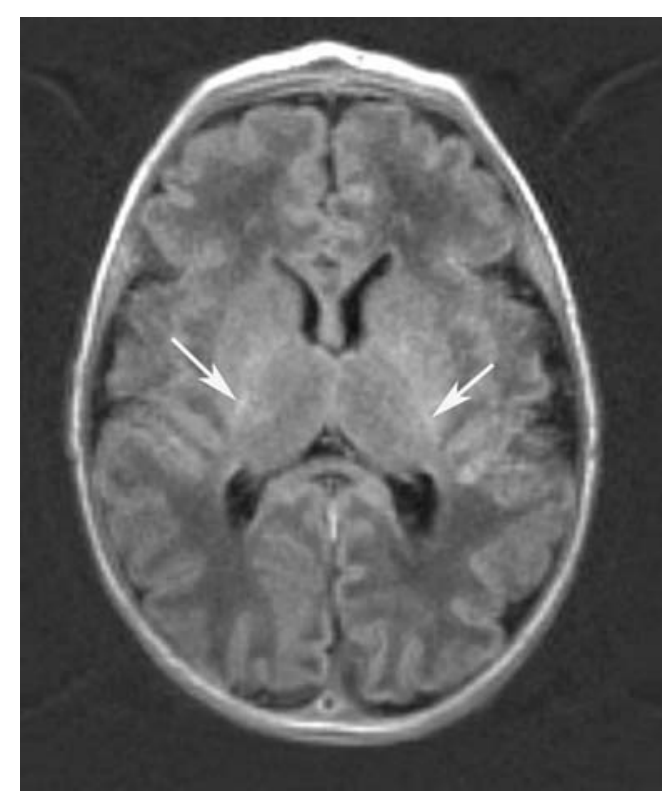

Fig. 3 Inversion T1-weighted transverse image at the level of the basal ganglia demonstrates an abnormally low level of myelinization of the posterior limb of the internal capsule (PLIC) (arrows) 
developed. At 1 year of age, neurological examination revealed intact cranial nerves, persistent axial and peripheral hypotonia, and no tremor. Developmental milestones were severely delayed for motor skills (no sitting nor standing), without apparent cognitive impairment. Exocrine pancreatic insufficiency manifested with chronic diarrhea and weight loss; oral enzymatic replacement was initiated. By the age of 15 months, the general condition of the patient worsened, with rapid neurological deterioration. After extensive discussion with the parents, supportive care was withdrawn and blood transfusions were stopped. The patient died at 18 months of life during sleep in a comfort care setting. Postmortem examination was declined.

\section{Neuroradiological analyses}

Magnetic resonance imaging and spectroscopy (MRI and MRS) scans were acquired on a 1.5-Tesla MR scanner in a multichannel head coil. Five-mm transverse slices were used for $\mathrm{T} 2$-weighted, inversion recovery $\mathrm{T} 1$-weighted, $\mathrm{T} 2 *$ weighted, and diffusion-weighted (b values of 0,500 , and 1,000 were used to calculate the ADC maps). MRS data was acquired in a multivoxel mode at an echo time of $135 \mathrm{~ms}$.

\section{Molecular genetic analyses}

DNA was extracted from leucocytes from blood and bone marrow with a commercial DNA extraction kit (Nucleon, Amersham). The deletion was identified using the multiplex ligation-dependent probe amplification (MLPA) P125 Mito probemix (MRC Holland Amsterdam, the Netherlands). The kit contains 31 different probes, which allows the estimation of the deletion size with an accuracy of $\pm 30 \mathrm{bp}$. Confirmation of the deletion was performed by Southern blot analysis using standard techniques (analysis performed in the Laboratory of Molecular Genetics, Division of Medical Genetics, Geneva University Hospital, Switzerland).

\section{Discussion}

Pearson marrow-pancreas syndrome (PS) is a fatal multisystemic mitochondrial disorder rarely diagnosed in the neonatal period. Onset is usually in early infancy, with sideroblastic anemia, neutropenia, thrombocytopenia, gastrointestinal symptoms, failure to thrive, metabolic acidosis with hyperlactic acidemia, and progressive liver and pancreatic failure $[1,5,8,10]$. The diagnosis of PS is strongly suspected when bone marrow aspirate shows the classical normal cellularity with vacuolization of marrow precursors.

This genetic disorder, caused by mitochondrial DNA (mtDNA) rearrangements, was first described by Pearson et al. in 1979 [7]. The tissue distribution and relative proportions of abnormal mtDNA gene products appear to determine the phenotype and clinical course $[4,9]$.

Although no "typical" presentation of PS exists, unexplained aregenerative anemia and failure to thrive with malabsorption in infancy are the most frequently reported symptoms. These non-specific symptoms may lead to extended work-up and delayed diagnosis. The early diagnosis of PS has been reported postnatally [3] and mostly in postmortem examinations of newborn infants whose death occurred after acute intractable metabolic acidosis and liver failure [1,8].

Our patient presented at birth with severe neonatal anemia, major hypotonia, and lactic acidosis, suggesting the diagnosis of PS. During his first year of life, he had a poor clinical course with multiple hospital admissions, and was treated conservatively. He developed failure to thrive with developmental delay. Exocrine pancreatic insufficiency occurred later, without diabetes. To our knowledge, this is the first report of such an early diagnosis of PS in a living child, and also the first description of encephalopathy and neuroradiological anomalies in a neonatal presentation of PS.

PS with neurological manifestations is well described in older children $[6,11]$. Neurological findings are either nonspecific, including mainly hypotonia, developmental delay, ataxia and tremor, or typical KSS. Our patient presented with an abnormal neurological status from birth. His symptoms were initially thought to reflect cerebral hypoxia resulting from the profound anemia, but a typical pattern of hypoxic-ischemic encephalopathy was not found on subsequent imaging (10 days of life). Therefore, a correlation between the abnormal neurological examination, abnormal MRI findings, and a mitochondrial disease was postulated. Note that non-specific neurological symptoms and MRI findings are described very often in PS [6, 11], but not at such an early age.

Lee et al. [6] described two extreme groups of patients with PS and neurological symptoms: one group with death before 4 years, the other with survival to later ages and the development of ataxia, tremor, KSS, or Leigh syndrome. Valanne et al. [11] reported cognitive impairment being the most common neurological symptom in PS survivors.

Brain MRI abnormalities have been described in a variety of mitochondrial disorders [2]. In our patient, brain MRI performed at term demonstrated early non-specific cerebral anomalies. Although brain imaging at this age is too early to represent the complete PS central nervous system involvement, similar findings are well described in previous reports. A broad range of images has been reported from normal findings to non-specific lesions, regardless of the clinical neurological course [6, 11]. Interestingly, the diffusely excessive high signal intensity seen in the white matter on T2-weighted images, with increased measurements of ADC values (Fig. 2), have also 
been described in children and adults with KSS/PS [11]. These MRI observations might be explained by a vacuolization involving the neuritic processes, which is thought to be the pathological process in mitochondrial deletion syndrome. Mitochondrial diseases are caused by defects in intracellular energy production. Tissues with high-energy requirements, like the brain, are extremely sensitive. PS patients have a large-scale mtDNA mutation range. MRI findings in PS with neurological involvement are well described. Although no direct genotype-phenotype correlation is known in PS, there is possibly a correlation between the degree of intracellular energy production impairment, the clinical neurological manifestations, and the corresponding imaging findings. The posterior limb of the internal capsule (PLIC) has also been described as frequently abnormal in metabolic diseases (Fig. 3). This may be the first objective manifestation of demyelinating lesions in the white matter in mitochondrial diseases [2]. Moreover, the lactate detected by spectroscopy in the posterior white matter (Fig. 1) is in favor of a pathogenetic role of the respiratory chain disturbance on the white matter changes. An elevation of brain lactate at MRS should raise the suspicion of a respiratory chain deficiency in the absence of hypoxia.

This case suggests that early neurological impairment, associated with non-specific brain MRI and MRS anomalies, could be the first neuropathologic expression of neonatal PS. In our case, it would have been interesting to perform follow-up brain MRI studies in order to analyze the disease progression and to better understand the correlation of imaging with the pathological neurological status. Furthermore, a detailed study of the heteroplasmy of the mtDNA deletion in different tissues would be relevant in the future for the genetic characterization of PS in relation to its clinical course and phenotypic expression. Unfortunately, the patient's poor general course lead to a conservative therapeutic approach that did not warrant unnecessary procedures. Moreover, the overwhelming diagnosis of PS worsened a fragile psychosocial situation, preventing the genetic screening of the mother to rule her out as a carrier.

Most cases described with abnormal MRI are older than our patient. This case is unique in terms of the precocity of the diagnosis, the association of clinical neurological manifestations, and the corresponding neuroimaging findings.

In conclusion, severe anemia with lactic acidosis and neurological impairment at birth should raise the suspicion of PS. The diagnosis may be confirmed quickly by bone marrow aspiration at the bedside and the genetic testing of peripheral blood to demonstrate the presence of mtDNA mutations. Brain MRI anomalies might be visualized in the neonatal period in PS. Follow-up brain MRI is mandatory to assess disease progression and the development of mitochondrial encephalopathy. A follow-up imaging at 6 months and 2 years of age would be recommended. However, PS with neurological involvement remains a rare entity with a variable spectrum of clinical and neuroradiological patterns.

Acknowledgments We thank M. Beck-Popovic, MD, for the hematological analyses, P. Wintermark, MD, for the brain MRI and MRS review, and J. Wagg, MD, PhD, and Prof. A. Moessinger, MD, for the review of the manuscript.

\section{References}

1. de Lonlay P, Fenneteau O, Touati G, Mignot C, Billette de Villemeur T, Rabier D, Blanche S, Ogier de Baulny H, Saudubray JM (2002) Hematologic manifestations of inborn errors of metabolism. Arch Pediatr 9:822-835

2. Elsås T, Rinck PA, Isaksen C, Nilsen G, Schjetne OB (1988) Cerebral nuclear magnetic resonance (MRI) in Kearns syndrome. Acta Ophthalmol (Copenh) 66:469-473

3. Giese A, Kirschner-Schwabe R, Blumchen K, Wronski L, Shalapour S, Prada J, Hernáiz Driever P, Brauer M, Schuelke M, Henze G, Seeger K (2007) Prenatal manifestation of pancytopenia in Pearson marrow-pancreas syndrome caused by a mitochondrial DNA deletion. Am J Med Genet A 143:285-288

4. Jacobs LJAM, Jongbloed RJE, Wijburg FA, de Klerk JBC, Geraedts JPM, Nijland JG, Scholte HR, de Coo IFM, Smeets HJM (2004) Pearson syndrome and the role of deletion dimers and duplications in the mtDNA. J Inherit Metab Dis 27:47-55

5. Knerr I, Metzler M, Niemeyer CM, Holter W, Gerecke A, Baumann I, Trollmann R, Repp R (2003) Hematologic features and clinical course of an infant with Pearson syndrome caused by a novel deletion of mitochondrial DNA. J Pediatr Hematol Oncol 25:948-951

6. Lee HF, Lee HJ, Chi CS, Tsai CR, Chang TK, Wang CJ (2007) The neurological evolution of Pearson syndrome: case report and literature review. Eur J Paediatr Neurol 11(4):208-214

7. Pearson HA, Lobel JS, Kocoshis SA, Naiman JL, Windmiller J, Lammi AT, Hoffman R, Marsh JC (1979) A new syndrome of refractory sideroblastic anemia with vacuolization of marrow precursors and exocrine pancreatic dysfunction. J Pediatr 95:976-984

8. Rötig A, Cormier V, Blanche S, Bonnefont JP, Ledeist F, Romero N, Schmitz J, Rustin P, Fischer A, Saudubray JM, Munnich A (1990) Pearson's marrow-pancreas syndrome. A multisystem mitochondrial disorder in infancy. J Clin Invest 86:1601-1608

9. Rötig A, Bourgeron T, Rustin P, Munnich A (1995) Phenotypic expression of mitochondrial genotypes in cultured skin fibroblasts and in Epstein-Barr virus-transformed lymphocytes in Pearson syndrome. Muscle Nerve 3:S159-S164

10. Superti-Furga A, Schoenle E, Tuchschmid P, Caduff R, Sabato V, DeMattia D, Gitzelmann R, Steinmann B (1993) Pearson bone marrow-pancreas syndrome with insulin-dependent diabetes, progressive renal tubulopathy, organic aciduria and elevated fetal haemoglobin caused by deletion and duplication of mitochondrial DNA. Eur J Pediatr 152:44-50

11. Valanne L, Ketonen L, Majander A, Suomalainen A, Pihko H (1998) Neuroradiologic findings in children with mitochondrial disorders. Am J Neuroradiol 19:369-377 